\title{
Lapurdum
}

Euskal ikerketen aldizkaria | Revue d'études basques |

Revista de estudios vascos | Basque studies review

$3 \mid 1998$

Numéro III

\section{Urkizu, P. : Zuberoako irri-teatroa. Recueil des farces} charivariques basques

Préface de J.B. Orpustan. Izpegi, St-Étienne-de-Baïgorry, 1998, 420p.

\section{Charles Videgain}

\section{(2) OpenEdition}

Journals

Édition électronique

URL : https://journals.openedition.org/lapurdum/1751

DOI : 10.4000/lapurdum. 1751

ISSN : 1965-0655

Éditeur

IKER

Édition imprimée

Date de publication : 1 octobre 1998

Pagination : 334-337

ISBN : 2-84127-152-8

ISSN : $1273-3830$

Référence électronique

Charles Videgain, «Urkizu, P. : Zuberoako irri-teatroa. Recueil des farces charivariques basques »,

Lapurdum [En ligne], 3 | 1998, mis en ligne le 01 septembre 2010, consulté le 24 février 2022. URL :

http://journals.openedition.org/lapurdum/1751 ; DOI : https://doi.org/10.4000/lapurdum. 1751 


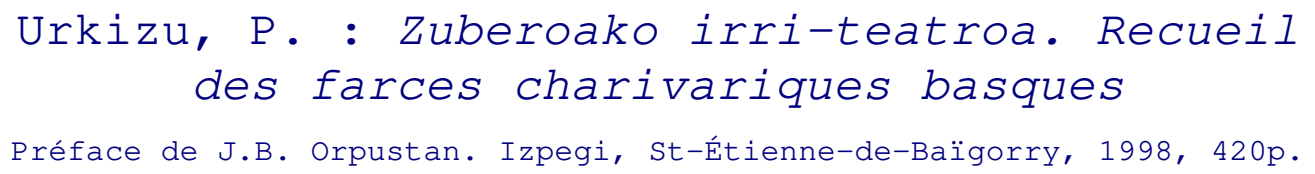

Patri Urkizu, professeur à l'UNED (Madrid), écrivain, est bien connu aussi pour son habileté à dénicher dans les bibliothèques ou les fonds particuliers peu accessibles des documents qu'il publie avec une louable régularité. On connaissait certes l'existence des textes qu'il publie ici, la plupart étant déposés dans les Bibliothèques Municipales de Bayonne et de Bordeaux. Cependant seuls quelques-uns d'entre eux avaient été peu ou prou mis à la disposition du public par G. Hérelle bien sûr, mais aussi G. Aresti, I. Mozos, Tx. Peillen et P. Urkizu lui-même.

On connaît le travail de Ch. Desplat: Le Charivari en Gascogne. La «morale des peuples »du XVIe au XXe siècle, Berger-Levrault, Paris, 1982, auquel il faut toujours se reporter pour comprendre la signification de ces formes de théâtre et de dénonciation. Les travaux de G. Hérelle restent encore indispensables pour mieux en connaître l'organisation. Mais c'est une lacune que vient opportunément combler P. Urkizu en donnant les textes des farces.

Après une introduction de J.B. Orpustan qui situe la farce comme genre littéraire dans son contexte historique dès l'Antiquité, P. Urkizu rappelle les diverses appellations en basque de ces farces charivariques : astolasterrak, 'courses d'ânes', tzintzarrotsak, galarrotsak, 'bruits de sonnailles', karrosak, thupina-usuak, tutak, trajeriak, toberak. Quant à l'origine des farces, P. Urkizu pense qu'elles existaient déjà en Pays Basque au XVIème siècle. L'anonymat des auteurs est la règle générale, ce qui ne signifie pas qu'il n'y ait pas eu d'auteur mais discrétion à leur égard, discrétion d'autant plus compréhensible que ces représentations pouvaient être l'objet de poursuites. On sait que le sujet qui provoquait ces farces et qui était représenté était généralement le remariage d'un veuf ou d'une veuve. Alors, souvent de nuit, tintamarre et envoi de lazzis et chansons permettaient de célébrer la farce. La victime pouvait mettre fin au tintamarre en offrant une somme d'argent ou des agapes à la jeunesse de la commune. L'infidélité conjugale pouvait aussi être le motif de ces farces. Il serait réducteur de n'y voir donc qu'un état de langue du dialecte souletin, même si j'y vois un remarquable corpus du point de vue dialetologique.

Les vers de ces textes d'une teneur dont la littérature basque semblait avare. Naturel et fraîcheur, verdeur aussi font le charme de ces documents essentiellement souletins mêlés de béarnais, de français, de castillan et de latin, le tout d'une grande créativité verbale dont le comique devait être bien rude pour les personnes égratignées mais irrésistible pour les acteurs et les spectateurs.

A titre d'exemple, voici la teneur d'une de ces pièces ; j'ai choisi Boubane eta Chilloberde, dont le manuscrit est conservé à la Bibliothèque Municipale de Bayonne. La pièce commence avec Boubane qui entame un monologue dans lequel il se plaint de son sort : paysan marié à une pendarde qui s'intéresse aux hommes jeunes qui la servent davantage qu'il ne le fait luimême, il est las d'élever les enfants d'autrui et de sa femme et de constater que des cornes lui poussent chaque jour. Arrive sa femme Chilloberde (litt. 'trou vert') qui l'invite à déjeuner avec le domestique Kapet que Boubane soupçonne de n'être point à jeun à aucun point de vue, ayant certainement déjeuné de lard et d'oeufs et d'une soupe au vin. Il se dispute avec Minika, autre domestique, puis interviennent les Satans dont Bulgifer qui dit son intention de conduire en enfer les deux époux, en persuadant Chilloberde que faire porter des cornes à son mari est du plus grand mérite en ce monde et en poussant Boubane à tuer sa femme. 
Jupiter approuve et Satan envoie Bulgifer et Jupiter accomplir cette tâche non sans les gratifier de quelques soufflets pour insubordination. Une bataille éclate entre eux, puis entre les domestiques. Minika est blessé par Kapet et secouru par Gaspar et Harburu qui le ramènent devant Chilloberde alors que Kapet nie les faits. Arrivent juge et greffier qui examinent les blessures de Minika et font le constat en français :

\author{
Il y a cinq cou de baton, \\ Trois pouce de longur, \\ Une pouce de largur, \\ Et un pouce de profondur, \\ Il a bien assez. \\ Pour mettre en prison \\ Il faut envoyer les gendarmes \\ Pour perte ce fripon.
}

Mandat d'amener est délivré contre Kapet. Nouvelle satanerie avec Bulgifer qui s'adresse aux jeunes filles de l'assistance et les invite pour le soir à lui ouvrir la porte. Boubane dit à Chilloberde qu'il veut la quitter mais elle l'assure qu'il sera bien obligé d'user ses habits à son côté. Satan suggère alors à Boubane d'étriper sa femme et le laisse en confiant à Bulgifer :

Gizon harek orai
Emaztia erho baleza
Harentako lizateke
Ifernüko leze beltxa.

C'est à dire : Si cet homme maintenant tuait sa femme, ce serait pour lui le gouffre noir de l'enfer.

Boubane se lamente de n'avoir pas «fréquenté » sa femme depuis dix mois : elle est enceinte pourtant de cinq mois ; il rappelle comment déjà il a dû aller chercher un jour la sage-femme mais qu'à leur retour, Chilloberde avait déjà accouché avec l'aide du... domestique. On assiste aussitôt à cette scène et quand Boubane arrive, Chilloberde lui donne un tout petit bout de boudin tandis qu'elle se remet de son accouchement en compagnie du domestique. Boubane veut la battre. Une autre satanerie montre alors Bulgifer et Jupiter invitant les jeunes filles à venir les voir, munies des œufs qu'elles ont volés à leurs mères pour faire bombance avec leurs amis.

Chilloberde conseille à Kapet d'aller voir l'huissier, chargé de cadeaux, pour qu'il les autorise à se marier ensemble. Mais le maire considère que Boubane n'est pas mort depuis assez longtemps pour que Chilloberde et Kapet puissent se marier sur-le-champ. Chilloberde part derechef voir le maire avec des cadeaux et le maire fait alors aussitôt publier les bans du mariage.

Ce résumé est trop rapide pour entrer dans les détails mais il suffira peut-être à laisser deviner les brusques changements de rythme, les ellipses et même les incohérences qui marquent le développement de la farce, ainsi que ce qu'elle suggère.

La langue est d'une extrême vivacité, les jeux de mots abondent et proverbes et expressions sont très habilement cousus dans le dialogue le plus cru. De toute manière, une étude fine de tous ces textes s'impose dorénavant pour en examiner les éléments très divers.

On l'a dit, les farces entrelardent diverses langues à travers le texte basque. A titre d'exemple voici comment Ricolord s'adresse à Charlemagne : 
Cailla ouste, Charlemagna,

Si nokieres enter bertat,

Ami parce que tous es

Una çambomba (p. 275).

On sait aussi le parti que tire la farce des vers macaroniques. Nous n'en donnerons que cet exemple :

\author{
Etés vous faché? \\ Que men fouti si non tabé, \\ C'est pourquoi jénipas, \\ Çoure acholiq bathere (p. 158).
}

Pour terminer, voici quelques observations sur le glossaire que P. Urkizu a eu l'excellente idée de fournir à la fin de son ouvrage. Le lecteur aurait préféré que la référence de chaque mot soit faite par rapport à une page plutôt que par un système d'abréviations malaisé qui ne permet pas par exemple de retrouver hasta. Aux mots traités, j'aurais ajouté l'expression haxeria artetik igaran zeren, littéralement 'le renard passa entre eux'pour 'ils se fâchèrent', 'ils se brouillèrent', expression encore fort courante aujourd'hui en Soule, et documentée ici dans un texte de 1907, p. 391.

Le lecteur remarquera que les verbes sont traduits en français sous leur forme au participe passé comme c'est le cas en basque. Dans le détail, karratü serait plutôt à traduire par 'rancir'que par 'rouiller'. Kuluna est à entendre comme radical verbal et ne peut être traduit par potrotaraino egon, l'idée principale ici étant celle de 'tromper', 'berner' ou 'couillonner' dans un registre plus marqué. A l'entrée lakha, remplacer 'demie'par 'demi'. Millok représente plutôt 'maïs'que 'sorgho', surtout dans le contexte, s'agissant de la 'méture'. Je doute que olhatü signifie ici 'adouci', dû sans doute à une lecture rapide de Lhande : je proposerais plutôt 'frapper', 'assener une volée de coups, une avoinée'et le contexte pousse à cette interprétation. Siflef devrait être porté comme zifleten entrée et non pas au sens de 'sifflet' mais de 'soufflet'. Susatu : il faut préciser que le mot s'applique en principe à la vache, d'où l'effet de grossièreté voulu par le texte. A l'entrée unkha, corriger 'hekto litro' par 'hektolitro' et préciser que 'conque' est une mesure. L'entrée xauxuma doit certainement être corrigée en xauxuna: d'une part, le terme n'est pas souletin, et le texte est en effet de Basse-Navarre ; d'autre part surtout, il ne signifie pas 'effronté' mais 'délicat', pris en mauvaise part, au sens de 'difficile à contenter' dont un synonyme pourrait être muturzuri ou le biscayen mizkin. Xikotatze est traduit par larru joite : le basque standard ne dispose-t-il que de cette expression pour traduire 'faire l'amour' ? Xaragoilla pose un problème : comme substantif, il signifie certes 'pantalon' mais dans le contexte (présence d'un auxiliaire second), xaragoilla est le radical verbal du verbe xaragoillatü, de sens érotique probable ici. Remplacer Sarohandy par Sarö̈handy, p. 92 ; Venu par Venus, Peti-Hun par Petik-Hun p. 419.

Ces menues remarques n'enlèvent rien à l'intérêt de ce travail. Il faut remercier P. Urkizu de cette nouvelle production, ainsi que la maison d'éditions Izpegi qui a su élaborer un ouvrage très soigné, pour une somme modique, à l'intention du public cultivé.

\title{
X. VIDEGAIN \\ UPPA UPRESA 5478 CNRS UPPA
}

